\title{
ALGUNAS DIMENSIONES CRÍTICAS EN EL ANÁLISIS DE LA GESTIÓN DE LAS POLÍTICAS EDUCATIVAS
}

\author{
Soc. Renato Opertti*
}

El análisis de la gestión de las políticas educativas en un sentido amplio se enmarca, por lo menos, en cinco aspectos que entendemos fundamentales para obtener una visión extensa e integrada que incorpora miradas externas e internas al sistema educativo.

En primer lugar, las políticas educativas establecen vínculos, ligazones y trabazones con los fines, modelos y estrategias de desarrollo y crecimiento que priman en un país en determinado contexto histórico y social ${ }^{1}$. Desde su propia génesis, está necesariamente incorporada a las dinámicas de desarrollo y por tanto no puede separarse de los entornos y sus influencias. Reconociendo las interacciones con los entornos, las políticas educativas se reflejan siempre en un presupuesto quinquenal de sueldos, gastos e inversiones, cuyo tiempo de duración es inferior al desarrollo progresivo de una política educativa.

Las tensiones entre los tiempos de maduración y concreción de las políticas educativas y los presupuestos quinquenales de gobierno es un asunto permanente que no nos debe llevar a encasillar caprichosamente las políticas educativas en el tiempo de administración de un gobierno. Por la propia naturaleza de la educación, los marcos de política tienen que estar pensados en una perspectiva de mediano a largo plazo, lo cual plantea dificultades de adaptación a la lógica presupuestal. Pero a la vez, se debe tener en cuenta que los sueldos, gastos e inversiones para iniciar, desarrollar y consolidar las políticas educativas, deben necesariamente reflejarse en el presupuesto, entendido como un instrumento crítico de la planificación técnico-financiera.

Dentro de este primer punto, quisiéramos también marcar la relación de las políticas educativas con otras políticas sociales. Como decíamos, la política educativa no puede independizarse de la discusión sobre las estrategias de desarrollo y crecimiento que se plantean; tampoco puede divorciarse de la consideración y el análisis de cómo influyen en ella otros aspectos sociales. El sistema educativo tiene, a nuestro criterio, una tendencia muy fuerte a la autorreferenciación, a ser muy endógeno en su análisis, con una capacidad limitada o disminuida de verse dentro de un contexto más general, que esencialmente tiene que ver con el hecho de que la política educativa constituye una condición necesaria para el logro de determinados niveles de bienestar, pero por sí misma no es suficiente.

La educación no puede, por sí sola, asumir la responsabilidad de la formación de la calidad de nuestros recursos humanos sin que se muevan, en el mismo sentido, otras piezas institucionales para que precisamente las políticas educativas puedan fortalecerse y crecer. La ejercitación del voluntarismo autárquico configura un acto de miopía cultural y social, que lleva muchas veces al desánimo, al fatalismo y a la resignación. Esta tendencia negativa de la autorreferenciación lleva a que la educación pueda verse a sí misma con relativa independencia de los contextos y de las instituciones que contribuyen a regular el comportamiento de una sociedad.

En segundo lugar, nos gustaría mencionar la propia formulación de las políticas educativas. ¿Cuál es el rol que tiene o que se le reserva a la planificación en la formulación de las políticas educativas? Partimos de la idea de que el planeamiento no es un conjunto de normas que expresan un deber $\operatorname{ser}^{2}$, pautado por el estricto cumplimiento de procesos y actividades desligadas de consideraciones e intervenciones sobre la diversidad de contextos que influyen en el quehacer educativo, sino más bien es la capacidad de articular una propuesta estratégica de conocimiento, de entendimiento y de actuación sobre realidades que a la vez son dadas y construidas. Esto no sólo implica tener una visión dinámica e 
interrelacionada de los elementos que conforman un sistema en estado de mutua dependencia, sino también entender que el planeamiento en un sentido estratégico cruza al sistema educativo en su conjunto, desde los niveles centrales hasta el centro educativo y las aulas. No podemos pensar que el planeamiento es propiedad del nivel central, porque en ese caso, trabajaríamos sobre un concepto timorato y mutilado de la acción de planificar, separando y fracturando las fases de diseño e implementación de las políticas educativas sin capacidad de retroalimentación crítica. Planificar incluye una secuencia lógica y sustantiva de acciones de diseño e implementación.

Nos preguntamos si este concepto de planeamiento está presente y de qué forma lo está, cuando se formulan políticas educativas. ¿Qué grado de precisión y alcance tienen las mismas? ¿En qué medida son expresiones de deseo, una necesidad transformada en objetivo general pero sin aterrizaje o presentan un desarrollo y una secuencia lógica que es consistente en todas las fases de su concreción? La formulación de políticas asimilables a una enunciación de buenas intenciones puede generar engaño y frustración en la medida que los objetivos generales no se desagregan en específicos; no se definen los contenidos de la política, las estrategias de implementación, su horizonte de tiempo así como tampoco sus costos. Estos últimos deben insertarse orgánicamente en el diseño de políticas para garantizar sustentabilidad técnica y financiera.

El conjunto de los aspectos reseñados hace en definitiva a la calidad técnica de las políticas formuladas, que se resume en la búsqueda de la intersección óptima entre cantidad y calidad del gasto. Ante todo, no es un problema de cantidad sino de objetivos en torno a los cuales trabajar y consensuar; después inexorablemente vendrá la discusión de los recursos necesarios para implementar esos objetivos. Si empezamos por discutir cantidad sin conceptualizar calidad, podemos aumentar los recursos pero corremos el riesgo cierto de no marcar diferencias en los resultados. Si nos planteamos, por ejemplo, un determinado objetivo de universalización de cobertura, nos debemos hacer la pregunta sobre cuánto cuesta en inversión de obra física, en equipamiento didáctico, en formación y capacitación de recursos humanos, entre otros aspectos relevantes. Estamos diciendo que la voluntad política de realizar objetivos y metas debe tener consistencia y sustento técnico-financiero, articulando cantidad y calidad.

En este marco, se genera una interrelación dinámica entre conceptos e instrumentos. En América Latina, en los últimos diez o quince años, se ha insistido reiteradamente en la aplicación de instrumentos que carecían, en algunos casos, de contenido o bien el mismo era débil o pobre -por ejemplo, la descentralización territorial y financiera sin sustento pedagógico, o la privatización de los servicios (subsidios a la demanda o la oferta) como forma "mágica" o única de mejorar el desempeño de los centros educativos ${ }^{3}$. Los modismos instrumentales han afectado a buena parte de los países de América Latina, y lo que se está revisando en la actualidad es la relación que debe trabarse entre los objetivos, los contenidos y los instrumentos -por ejemplo, en el caso de la descentralización- para efectivamente mejorar la gestión.

En tercer lugar, nos preguntamos sobre las supuestas claves del consenso y la legitimidad de las políticas educativas. Hay una tensión muy fuerte, que permea el sistema educativo en su conjunto, entre la verticalidad de las relaciones y la horizontalidad de las discusiones. Cuando discutimos las políticas educativas en los diferentes niveles del sistema, ¿prima la horizontalidad en la discusión entre un inspector y un director, o entre éstos últimos y los docentes o entre el cuerpo directivo y los mandos medios? ¿Estamos acostumbrados culturalmente a admitir visiones e interpretaciones distintas a las que abrigamos y a asumir que la horizontalidad de la discusión es un espacio de legitimación del consenso de la política educativa? ¿O estamos acostumbrados a que se nos diga "esto es así" y contestamos "bien, acatamos" o disentimos y "nos rehusamos a discutir"? ¿Cuáles son, en definitiva, las bases necesarias para generar una cultura de la discusión horizontal en el sistema educativo? No se trata únicamente de ambientar espacios saludables de contrastes de perspectivas y de consolidación de la pluralidad en la construcción colectiva, sino también de reconocer que el poder está presente en la diversidad de relaciones que se dan en el sistema educativo y que por tanto, no es únicamente un problema cupular. 
El desafío principal radica en superar el precepto "hay que bajar línea" y comprometernos sí en el armado colectivo de la política educativa, favoreciendo la horizontalidad de la discusión sin caer en un esquema ingenuo de participación retórica.

¿Cómo nos comprometemos y nos involucramos con lo que sentimos que es una buena decisión de política educativa? Si pensamos en determinados objetivos generales de política educativa -mejorar la calidad de los aprendizajes y aumentar la equidad social-, seguramente nos pondremos rápidamente de acuerdo; el tema central es cómo los concretamos o bien cómo pactamos el disenso para el desarrollo de los mismos. Asimismo, nos debemos también interrogar sobre las características y los contenidos de la comunicación para situarla en un marco amplio de análisis, evitando el predominio de posturas corporativas con el síndrome del interés afectado. Si no reflexionamos ni desarrollamos los espacios y los márgenes para los acuerdos y los disensos, la concreción de las políticas educativas se verá severamente afectada. Tampoco la comunicación es un problema únicamente cupular.

En cuarto lugar, nos interrogamos sobre la propia gestión de las políticas educativas o visto en una perspectiva más amplia, sobre la necesaria integración entre las fases de diseño e implementación. A nuestro criterio, se comete un grave error si apostamos a su nítida separación, esgrimiendo que hay roles bien diferenciados de diseñadores e implementadores. La evaluación continua es fundamental como elemento unificador de ambas fases, buscando aunar una visión que deriva de un nivel central y que se va progresivamente construyendo y transformando conforme se acerca a su destino final - las aulas - ya sea como actividad, resultado o impacto.

Otra forma de separación que causa problemas, es el eje técnico/administrativo. La distinción radical entre una esfera de acción administrativa "rutinaria y poco productiva" y una pedagógica-didáctica conspira contra la asunción de roles de gerenciamiento de las instituciones educativas. No se visualiza adecuadamente que la realidad es un todo cuyo desmembramiento permite identificar y priorizar áreas de intervención, pero en modo alguno sustituye la visión de conjunto que se debe tener. Lo que importa finalmente es que un buen gerenciamiento constituya un medio adecuado para incidir positivamente en mejoras de aprendizajes socialmente distribuidos de forma más pareja. Precisamente por no tener muchas veces una perspectiva del todo en su conjunto, terminamos por fragmentar e inundar la realidad con acciones parcializadas y bajos resultados, distanciando la gestión institucional de la pedagógica. El manejo de la diversidad de elementos implicados en la gestión, es un activo que debe ser aprovechado para incidir de manera más eficiente y más eficaz.

Bajo el supuesto de entender la necesidad de integrar y tener en cuenta múltiples aspectos, la gestión de las políticas educativas debería pasar por jerarquizar el centro educativo como espacio estructurador de la gestión, y de la construcción de los procesos y actividades implicadas para lograr optimizarla. Los centros educativos no son islas en la cual desembarcan insumos no ambientados en políticas educativas, sino que configuran el eje canalizador de las acciones que el sistema planifica en su conjunto.

Por último, nos gustaría referirnos a dos aspectos metodológicos que están presentes en los procesos de gestión de las políticas educativas. Primeramente, es necesario entender que al igual que asignamos relevancia a los condicionamientos de tipo estructural en las conductas y resultados, también debemos poner énfasis en el mundo de las expectativas y las aspiraciones de la diversidad de actores implicados en el quehacer educativo -por ejemplo, jóvenes y padres-. En los estudios realizados a nivel del Ciclo Básico de Educación por el Programa MESyFOD (Modernización de la Educación Secundaria y la Formación Docente), se ha observado que cuando los padres y los hijos comparten un proyecto educativo común, esto es, cuando ambos tienen las mismas aspiraciones sobre el máximo de instrucción que se espera alcanzar, los rendimientos en las pruebas censales de aprendizaje en los $3^{\text {eros }}$ Años de Ciclo Básico son más altos que cuando hay disenso familiar ${ }^{4}$. 
Hay todo un espacio de trabajo sobre el mundo de las expectativas, en la relación familias jóvenes- educación que no puede pasar inadvertida en la consideración de la gestión de las políticas educativas. Debemos, en muchos casos, superar la visión determinista de creer que todo viene de afuera, impuesto, y que en consecuencia, nuestra capacidad de respuesta es casi nula, resignándonos al fatalismo o a la propia lógica de los hechos. Nosotros también podemos -como sujetos actuantes-, intervenir activamente en modificar las situaciones, si abrigamos una visión dinámica e interactiva de la realidad.

Asimismo, debemos también intentar superar las brechas y las discrepancias entre un discurso teórico progresista y una práctica retrógrada, lo cual lleva a la perversa consolidación del "doble discurso" que tanto criticamos legítimamente. En gran medida, se trata de realizar la auto-crítica y ver cuán distantes o cuán cercanas están dichas prácticas de nuestros conceptos guías. En definitiva, es parte sustantiva del sinceramiento ético e intelectual.

\section{Notas:}

${ }^{1}$ La relación entre educación y desarrollo, vista desde una perspectiva regional latinoamericana, puede consultarse, por ejemplo:

\section{UNESCO/CEPAL/PNUD.}

Nassif, R., Rama, G. y Tedesco, J . 1984. El sistema educativo en América Latina. Educación y democracia. En Serie Educación y Sociedad. págs. 105-13. Buenos Aires: Kapeluz.

PNUD/Educación. Gómez Buendía, H. (director). 1998. La agenda del siglo XXI. Hacia un desarrollo humano. En Concepciones de estado y educación. Capítulo 4. págs. 119-156. Colombia: Tercer Mundo Editores.

Desde una perspectiva nacional, pueden consultarse por ejemplo:

Rama, G. 1987. La democracia en Uruguay. Una perspectiva de interpretación. Buenos Aires: Grupo Editor Latinoamericano.

ANEP, Rama, G. (dirección técnica general) y Opertti, R. (supervisor técnico y redactor principal). 2000. Una visión integral del proceso de reforma educativa en Uruguay. En Educación y sociedad, una visión del pasado que desafía al presente y al futuro. 1995-1999. Capítulo I. págs. 21-26. Montevideo: Impresores Asociados / Fotosistemas S.A.

${ }^{2}$ Las diferentes dimensiones de la planificación son analizadas en:

Ander_Egg, E. 1996. La Planificación Educativa. Conceptos, métodos, estrategias y técnicas para educadores. págs. 13-4. Buenos Aires: Magisterio del Río de la Plata.

La evolución de los procesos de planeamiento educativo en la segunda mitad del siglo XX pueden consultarse en:

Magnen, A. 1990. Los proyectos de educación: preparación, financiación y gestión. En UNESCO, Instituto Internacional de Planeamiento de la Educación. págs. 7-28. París: UNESCO. 
${ }^{3}$ Para una discusión de estos temas, véase por ejemplo:

BID. 1996. ¿Qué rumbo debe tomar el mejoramiento de la Educación en América Latina?. En Seminario sobre Reforma Educativa. Buenos Aires: BID.

McEwan. Patrick, J. y Carnoy, M. 1999. The Effectiveness and Efficiency of Private Schools in Chile Voucher System. En School of Education. Stanford University. California.

CEPAL, División de Desarrollo Social. 2000. ¿Hacia donde va el gasto público en educación? Logros y desafíos. En Serie Políticas Sociales. Volumen II. Reformas sectoriales y grupos de interés. Santiago de Chile: CEPAL.

CEPAL, Franco, R. (coord.), Opertti, R. y De Armas, G. 2001. Reflexiones sobre la Reforma Educativa en Uruguay (1995-1999). En Sociología del Desarrollo, Políticas Sociales y Democracia. págs. 312322. México: XXI.

CEPAL, Franco, R. (coord.), Cosse, G. 2001. El sistema de voucher educativo: una nueva y discutible panacea para América Latina. En Sociología del Desarrollo, Políticas Sociales y Democracia. págs. 289-3. México: XXI.

${ }^{4}$ El censo de aprendizajes se aplicó en las áreas de Matemática, Lengua Materna, Ciencias Experimentales y Ciencias Sociales en los $3^{\text {eros }}$ Años de Ciclo Básico de Educación Media (año 1999) en liceos públicos y privados así como en escuelas técnicas. Sus principales características y resultados pueden consultarse en MESyFOD, ANEP / Primera comunicación de resultados. Montevideo. MESyFOD/ANEP. 2000. Sobre la incidencia del consenso familiar en los resultados, puede consultarse MESyFOD. ANEP/ Formación de actitudes y opiniones: los estudios desde la perspectiva de los estudiantes. Séptima comunicación. Montevideo. MESyFOD/ANEP. 2000.

*Coordinador General, Unidad Ejecutora de los Programas de Educación Media y Formación Docente, MES y FOD, Programa de Modernización de la Educación Secundaria y la Formación Docente; UTU/ BID y Programa de Fortalecimiento de la Educación Técnica, ANEP. Docente e Investigador, Universidad ORT Uruguay. 